\title{
Drug survival of tumor necrosis factor $\alpha$ inhibitors in patients with ankylosing spondylitis in Korea
}

\author{
Hyemin Jeong ${ }^{1}$, Yeong Hee Eun ${ }^{1}$, In Young Kim ${ }^{1}$, Hyungjin Kim ${ }^{1}$, Joong Kyong Ahn², Jaejoon Lee \\ Eun-Mi Koh ${ }^{1}$, and Hoon-Suk Cha ${ }^{1}$
}

${ }^{1}$ Department of Medicine, Samsung Medical Center, Sungkyunkwan University School of Medicine, Seoul; ${ }^{2}$ Department of Medicine, Kangbuk Samsung Hospital, Sungkyunkwan University School of Medicine, Seoul, Korea
Background/Aims: To evaluate drug survival of the tumor necrosis factor $\alpha$ inhibitors (TNFi) and risk factors for the drug discontinuation in patients with ankylosing spondylitis (AS).

Methods: We retrospectively evaluated $487 \mathrm{AS}$ patients at a single tertiary hospital. Among the TNFi users, drug survival and risk factors of TNFi discontinuation were investigated.

Results: Among 487 patients, 128 AS patients were treated with at least one TNFi. Patients who were treated with TNFi were younger at disease onset, had more peripheral manifestations, and had higher level of acute phase reactants and body mass index than those of TNFi non-users at baseline. Of 128 patients, 28 patients (21.9\%) discontinued first TNFi therapy during the follow-up period of $65.1 \pm 27.9$ months. In the multivariable analysis, female (hazard ratio [HR], 6.08; 95\% confidence interval [CI], 2.27 to 16.27; $p=0.003$ ), hip involvement (HR, 2.52; 95\% CI, 1.08 to $5.87 ; p=0.033$ ) and a high C-reactive protein (CRP; HR, 1.10; 95\% CI, 1.00 to 1.21; $p=0.044)$ were risk factors for drug discontinuation. Etanercept showed better survival rate than infliximab. The main reason for discontinuation of TNFi was inefficacy.

Conclusions: TNFi discontinuation rate of Korean patients with AS seems to be similar to those with the European patients. Female sex, hip involvement, CRP, and the type of TNFi were associated with TNFi discontinuation.

Keywords: Spondylitis, ankylosing; Tumor necrosis factor-alpha; Survival; Risk factors

\section{INTRODUCTION}

Ankylosing spondylitis (AS) is a chronic inflammatory disease that primarily affects the sacroiliac joints and spine. Tumor necrosis factor $\alpha$ (TNF- $\alpha$ ) is important cytokine in the disease process of AS as previous studies found abundant levels of TNF- $\alpha$ in the sacroiliac joint of AS patients $[1,2]$. TNF- $\alpha$ inhibitors (TNFi), infliximab, etanercept, and adalimumab, have proved to be effective treatment options for patients with active AS [3-5]. Ac- cording to the meta-analysis, etanercept, adalimumab, and infliximab showed similar effects on reducing signs and symptoms of AS [6]. However, these TNFi have different structure, mechanism of action, mode of administration, and half-life in the body. Patients who failed one drug may benefit from another TNFi.

Drug survival for spondyloarthritis is longer than that for rheumatoid arthritis (RA) [7]. Although TNFi is effective in the treatment of AS, some AS patients cannot continue TNFi for several reasons. They may not respond 
properly to the TNFi and others experience side effects related to the drugs. Several biologic agents other than TNFi are also available for patients with RA, while TNFi is the only biologic agent used in Korea for patients with AS currently. Studies about factors for drug survival for patients with AS have not sufficient as compared with RA patients and only a few studies are available for the Asian population. We wished to investigate the use of TNFi for AS patients under real-world circumstances. The aims of this study were to evaluate drug survival of the TNFi in AS patients and determine the risk factors for discontinuation of TNFi in patients with AS.

\section{METHODS}

\section{Study population and parameters collected}

The subjects were selected in a consecutive manner from a pool of patients visiting the rheumatology outpatient clinic of Samsung Medical Center in Seoul, South Korea between January 2001 and January 2011. We selected patients who were diagnosed with AS by a rheumatologist and fulfilled the modified New York criteria [8]. One patient who used golimumab as a first TNFi was excluded due to representing a small number. The approval of golimumab for the treatment of AS was much later than that of the other TNFi in Korea. In addition, patients whose follow-up duration of TNFi, namely the time from the initiation of first TNFi to the last visit to the outpatient clinic, was less than 24 months were excluded from the study. In this study population, TNFi were prescribed from September 2005 to July 2013. A total of 487 patients were included and their medical records were reviewed retrospectively. The clinical characteristics at baseline were collected, including Creactive protein (CRP), erythrocyte sedimentation ratio (ESR), HLA (human leukocyte antigen)-B27 positivity, body mass index (BMI), smoking status, and X-rays of the spine and pelvis. Peripheral arthritis included the knee, ankle, foot, elbow, wrist, and hand joints. Hip involvement was defined according to the clinical opinion of the rheumatologist, such as pain and limited range of motion and radiographic evidence of hip arthritis, defined by at least the score of 1 in the Bath Ankylosing Spondylitis Radiology Hip Index (BASRI-h) scoring system [9]. Disease duration was defined as the time from the symptom onset to the first visit to the outpatient clinic. Two trained rheumatologists (H.J. and I.Y.K.) assessed the radiographs at baseline. The sacroiliitis grade was defined as the sum of each side of the sacroiliitis grade according to the modified New York criteria [8]. The presence of new syndesmophytes was also assessed. The intra-class correlation coefficient of the status score for two readers was 0.87 (95\% confidence interval [CI], 0.65 to 0.95$)$.

\section{Biologics and outcome}

Three TNFi were used for the treatment of AS: etanercept, adalimumab, and infliximab. Biologics were administered according to the standard protocol recommended by the manufacturer. At the time of initiation of the TNFi, all patients had Bath Ankylosing Spondylitis Disease Activity Index (BASDAI) greater than four, this was in spite of prior treatment with at least two different nonsteroidal anti-inflammatory drugs or sulfasalazine for more than 3 months according to the standard of Korean Health Insurance Review and Assessment Service. The primary outcome was discontinuation of the first TNFi therapy due to any cause except for remission. Remission was defined according to the treating rheumatologist's assessment of disease activity rather than been strictly defined; for example, by Ankylosing Spondylitis Disease Activity Score (ASDAS). Secondary outcome was to assess the predictors of discontinuation and drug survival. Drug survival was calculated as the number of months between the dates of the first start and last day of the drug prescription. Reason for discontinuation of TNFi was also assessed. Insufficient efficacy was characterized as primary (never achieved meaningful response) and secondary failure (achieved meaningful response, then lost response during therapy).

This study was approved through the Institutional Review Board of the Samsung Medical Center.

\section{Statistical analysis}

Descriptive statistics were used to identify the characteristics of the study population. Categorical variables were compared between groups using the chi-square test or Fisher exact test. For continuous variables, the data are presented as the mean $\pm \mathrm{SD}$, and variables were compared using $t$ test or one way analysis of variance analysis. Univariate and multivariate Cox regression analysis 
Table 1. Clinical characteristics of ankylosing spondylitis patients between TNFi user and non-user at baseline

\begin{tabular}{|c|c|c|c|}
\hline Characteristic & TNFi non-user $(\mathrm{n}=359)$ & TNFi user $(n=128)$ & $p$ value \\
\hline Age at disease onset, yr & $26.4 \pm 8.9$ & $24.4 \pm 9.9$ & 0.032 \\
\hline Female sex & $64(17.8)$ & $16(12.5)$ & 0.163 \\
\hline Disease duration, mon & $75 \cdot 5 \pm 85.3$ & $72.8 \pm 81.1$ & 0.756 \\
\hline Peripheral arthritis & $73(20.3)$ & $48(37.5)$ & $<0.001$ \\
\hline Hip arthritis & $31(8.6)$ & $28(21.9)$ & $<0.001$ \\
\hline Enthesitis & $27(7 \cdot 5)$ & $22(17.2)$ & 0.002 \\
\hline Uveitis ever & $73(20.3)$ & $28(21.9)$ & 0.712 \\
\hline Syndesmophytes & $90(25.1)$ & $33(25.8)$ & 0.886 \\
\hline Sacroiliitis grade ${ }^{a}$ & $4.8 \pm 1.6$ & $4.9 \pm 1.7$ & 0.496 \\
\hline ESR at baseline, $\mathrm{mm} / \mathrm{hr}$ & $33.0 \pm 25.0$ & $51.9 \pm 33.6$ & $<0.001$ \\
\hline CRP at baseline, mg/dL & $1.71 \pm 2.4$ & $2.9 \pm 3.2$ & $<0.001$ \\
\hline HLA-B27 positive $(n=365)$ & $248 / 268(92.5)$ & $89 / 97(91.8)$ & 0.803 \\
\hline BMI, $\mathrm{kg} / \mathrm{m}^{2}$ & $23.9 \pm 3.3$ & $24.8 \pm 3.8$ & 0.012 \\
\hline Hypertension & $44(12.3)$ & $21(16.4)$ & 0.236 \\
\hline Diabetes & $12(3 \cdot 3)$ & $7(5 \cdot 5)$ & 0.286 \\
\hline Dyslipidemia & $27(7.5)$ & $20(15.6)$ & 0.008 \\
\hline Ischemic heart disease & $1(0.3)$ & $2(1.6)$ & 0.170 \\
\hline Current smoker & $32(8.9)$ & $17(13.3)$ & 0.172 \\
\hline
\end{tabular}

Values are presented as mean \pm SD or number $(\%)$.

TNFi, tumor necrosis factor $\alpha$ inhibitor; ESR, erythrocyte sediment rate; CRP, C-reactive protein; HLA, human leukocyte antigen; BMI, body mass index.

${ }^{a}$ The sacroiliitis grade refers to the sum of each side of the sacroiliitis grade according to the modified New York criteria.

with hazard ratios (HRs) were used for the identification of factors associated with drug discontinuation of the first TNFi. The variables associated with $p$ value outcomes $\leq 0.20$ were included in the multivariate analysis. Kaplan-Meier analysis was used to visualize drug specific survival and log-rank test was used to compare the distributions. Adjusted and unadjusted HRs were estimated for infliximab verses etanercept, adalimumab versus etanercept, and infliximab versus adalimumab. $p$ values were corrected by Scheffe's method due to multiple testing. All analyses were performed using SPSS version 19.0 (IBM Co., Armonk, NY, USA) and SAS version 9.4 (SAS Institute Inc., Cary, NC, USA). Statistical significance was assigned when $p$ values were $<0.05$.

\section{RESULTS}

\section{Characteristics of TNFi user compared with TNFi non-user}

Of the 487 patients with AS, 128 patients were started on a TNFi during the follow-up period. Mean follow-up duration of 487 patients was $72.6 \pm 52.5$ months. The baseline clinical characteristics of the patients with AS according to the TNFi use are presented in Table 1. Patients who started on TNFi during the follow-up period were younger at the disease onset, had more peripheral manifestations, and showed higher level of acute phase reactants than TNFi non-users at baseline. Interestingly, TNFi users had a higher BMI and prevalence of dyslipidemia than patients who were TNFi non-users.

\section{Treatment course and characteristics for the TNFi user}

Among the 128 study patients, 57 patients (44.5\%) started on etanercept, 56 (43.8\%) on adalimumab with 15 (11.7\%) on infliximab as a first-choice treatment. Mean follow-up duration, from the time of TNFi therapy start to the last visit of outpatient clinic, was $65.1 \pm 27.9$ months. Of the 128 patients treated with their first TNFi, 25 of them (19.5\%) switched to a second TNFi after discontinuing the first TNFi and 98 patients $(76.6 \%)$ remained 
Table 2. Patient characteristics at the time of initiation of first TNFi in patients with ankylosing spondylitis

\begin{tabular}{|c|c|c|c|c|}
\hline Characteristic & Etanercept $(\mathrm{n}=57)$ & Adalimumab $(\mathrm{n}=56)$ & Infliximab (n = 15) & $p$ value \\
\hline Age at disease onset, yr & $26.0 \pm 11.6$ & $23.5 \pm 8.5$ & $21.8 \pm 6.7$ & 0.239 \\
\hline Female sex & $10(17 \cdot 5)$ & $4(7.1)$ & $2(13 \cdot 3)$ & 0.263 \\
\hline Disease duration, mon & $71.6 \pm 87.4$ & $77.1 \pm 79.8$ & $61.5 \pm 61.8$ & 0.796 \\
\hline Peripheral arthritis, except hip & $23(40.4)$ & $20(35 \cdot 7)$ & $5(33 \cdot 3)$ & 0.841 \\
\hline Hip arthritis & $11(19 \cdot 3)$ & $12(21.4)$ & $5(33 \cdot 3)$ & 0.447 \\
\hline Enthesitis & $9(15.8)$ & $9(16.1)$ & $4(26.7)$ & 0.542 \\
\hline Uveitis ever & $10(17.5)$ & $14(25 \cdot 0)$ & $4(26.7)$ & 0.543 \\
\hline Inflammatory bowel disease & o & $2(3.6)$ & $1(6.7)$ & 0.104 \\
\hline Syndesmophytes & $17(29.8)$ & $15(26.8)$ & $1(6.7)$ & 0.199 \\
\hline Sacroiliitis grade ${ }^{a}$ & $5.1 \pm 1.8$ & $5.0 \pm 1.7$ & $4 \cdot 3 \pm 1.1$ & 0.298 \\
\hline ESR at TNFi start, $\mathrm{mm} / \mathrm{hr}$ & $34 \cdot 3 \pm 12.8$ & $33.0 \pm 10.2$ & $28.5 \pm 10.2$ & 0.370 \\
\hline CRP at TNFi start, mg/dL & $2.7 \pm 3.2$ & $2.8 \pm 3 \cdot 3$ & $2.9 \pm 2.8$ & 0.970 \\
\hline HLA-B27 positive $(\mathrm{n}=97)$ & $43 / 45(95 \cdot 6)$ & $38 / 41(92.7)$ & $8 / 11(72.7)$ & 0.051 \\
\hline $\mathrm{BMI}, \mathrm{kg} / \mathrm{m}^{2}$ & $24.2 \pm 3.6$ & $25.6 \pm 3.7$ & $24 \cdot 4 \pm 4 \cdot 4$ & 0.162 \\
\hline Hypertension & $7(12.3)$ & $10(19.7)$ & $4(26.7)$ & 0.320 \\
\hline Diabetes & $4(7.0)$ & $2(3.6)$ & $1(6.7)$ & 0.642 \\
\hline Dyslipidemia & $9(15.8)$ & $8(14 \cdot 3)$ & $3(20.0)$ & 0.835 \\
\hline Ischemic heart disease & 0 & $2(3.6)$ & 0 & 0.411 \\
\hline Current smoker & $5(8.8)$ & $10(17 \cdot 9)$ & $2(13 \cdot 3)$ & 0.400 \\
\hline BASDAI & $9.1 \pm 1.1$ & $9.5 \pm 0.6$ & $9.5 \pm 0.6$ & 0.016 \\
\hline NSAID & $57(100)$ & $56(100)$ & $14(93 \cdot 3)$ & 0.117 \\
\hline Prednisolone & $9(15.8)$ & $10(17.9)$ & $5(33 \cdot 3)$ & 0.292 \\
\hline Sulfasalazine & $49(86.0)$ & $48(85.7)$ & $15(100)$ & 0.356 \\
\hline Methotrexate & $12(21.1)$ & $3(5.4)$ & $1(6.7)$ & 0.035 \\
\hline
\end{tabular}

Values are presented as mean \pm SD or number (\%).

TNFi, tumor necrosis factor $\alpha$ inhibitor; ESR, erythrocyte sediment rate; CRP, C-reactive protein; HLA, human leukocyte antigen; BMI, body mass index; BASDAI, Bath Ankylosing Spondylitis Disease Activity Index; NSAID, nonsteroidal anti-inflammatory drug.

${ }^{a}$ The sacroiliitis grade refers to the sum of each side of the sacroiliitis grade according to the modified New York criteria.

on their first TNFi during the follow-up period. For five patients (3.9\%), they stopped the first TNFi without starting on a second TNFi. Of these five patients, two patients discontinued due to remission. For the three patients, they discontinued the drug due to insufficient efficacy or side effect and these three patients did not want to use a second TNFi. Therefore, a total of 28 patients $(21.9 \%)$ were classified as discontinuation of the first TNFi therapy and the remaining 100 patients were classified as continuation. Clinical characteristics of the patients at the time of initiation of first TNFi are presented in Table 2. Patients who started etanercept as a first TNFi had lower BASDAI and were prescribed methotrexate more frequently at the time of the initiation of TNFi. Other clinical characteristics were not different between groups.

\section{Predictors of discontinuation}

The mean drug survival of first TNFi was $55.1 \pm 33.3$ months. Clinical characteristics between patients with continuation and discontinuation of the first TNFi are presented in Table 3. Female sex, presence of hip arthritis, high CRP, and type of TNFi were significantly different between two groups. In the multivariable analysis, 
Table 3. Clinical characteristics between the patients with continuation and discontinuation of first TNFi at the time of the first TNFi initiation

\begin{tabular}{|c|c|c|c|c|}
\hline Variable & $\begin{array}{l}\text { Continuation } \\
\qquad(\mathrm{n}=100)\end{array}$ & $\begin{array}{l}\text { Discontinuation } \\
\qquad(\mathrm{n}=28)\end{array}$ & OR $(95 \% \mathrm{CI})$ & $p$ value \\
\hline Age at disease onset, yr & $24.6 \pm 10.4$ & $23.6 \pm 8.0$ & $0.99(0.95-1.03)$ & 0.616 \\
\hline Age at TNFi start & $33.5 \pm 11.7$ & $31.5 \pm 10.8$ & $0.98(0.95-1.02)$ & 0.417 \\
\hline Female sex & $9(9.0)$ & $7(25.0)$ & $3.37(1.13-10.08)$ & 0.030 \\
\hline Disease duration, mon & $72.1 \pm 81.6$ & $75.3 \pm 80.7$ & $1.00(0.99-1.01)$ & 0.853 \\
\hline Peripheral arthritis, except hip & $35(35.0)$ & $13(46.4)$ & $1.61(0.69-3.76)$ & 0.272 \\
\hline Hip arthritis & $16(16.0)$ & $12(42.9)$ & $3.94(1.57-9.88)$ & 0.003 \\
\hline Enthesitis & $18(18.0)$ & $4(14 \cdot 3)$ & $0.76(0.23-2.46)$ & 0.646 \\
\hline Uveitis ever & $25(25.0)$ & $3(10.7)$ & $0.36(0.10-1.30)$ & 0.118 \\
\hline Syndesmophytes & $28(28.0)$ & $5(17.9)$ & $0.56(0.19-1.62)$ & 0.283 \\
\hline Sacroiliitis grade ${ }^{\mathrm{a}}$ & $5.0 \pm 1.8$ & $4.7 \pm 1.5$ & $0.87(0.66-1.14)$ & 0.301 \\
\hline ESR at TNFi start, $\mathrm{mm} / \mathrm{hr}$ & $46.4 \pm 30.9$ & $52.1 \pm 35.4$ & $1.01(0.99-1.02)$ & 0.403 \\
\hline CRP at TNFi start, mg/dL & $2.4 \pm 2.7$ & $4 \cdot 1 \pm 4 \cdot 3$ & $1.15(1.02-1.30)$ & 0.022 \\
\hline HLA-B27 positive $(\mathrm{n}=97)$ & $73 / 78(93.6)$ & $16 / 19(84.2)$ & $0.37(0.08-1.69)$ & 0.197 \\
\hline BMI, $\mathrm{kg} / \mathrm{m}^{2}$ & $24.8 \pm 3.2$ & $25 \cdot 0 \pm 5 \cdot 3$ & $1.01(0.91-1.13)$ & 0.850 \\
\hline Hypertension & $16(16.0)$ & $5(17.9)$ & $1.14(0.38-3.45)$ & 0.815 \\
\hline Diabetes & $4(4 \cdot 0)$ & $3(10.7)$ & $2.88(0.61-13.71)$ & 0.184 \\
\hline Dyslipidemia & $17(17.0)$ & $3(10.7)$ & $0.59(0.16-2.16)$ & 0.422 \\
\hline Current smoker & $11(11.0)$ & $6(21.4)$ & $2.21(0.74-6.62)$ & $0.15^{8}$ \\
\hline BASDAI & $9.3 \pm 0.9$ & $9.3 \pm 1.0$ & $0.96(0.60-1.53)$ & 0.859 \\
\hline Prednisolone & $18(18.0)$ & $6(21.4)$ & $1.24(0.44-3.50)$ & 0.682 \\
\hline Sulfasalazine & $87(87.0)$ & $25(89 \cdot 3)$ & $1.25(0.33-4.72)$ & 0.747 \\
\hline Methotrexate & $13(13.0)$ & $3(10.7)$ & $0.80(0.21-3.04)$ & 0.747 \\
\hline Etanercept & $50(50.0)$ & $7(25.0)$ & $0.33(0.13-0.85)$ & 0.022 \\
\hline Adalimumab & $42(42.0)$ & $14(50.0)$ & $1.38(0.60-3.20)$ & 0.452 \\
\hline Infliximab & $8(8.0)$ & $7(25.0)$ & $3.83(1.25-11.75)$ & 0.019 \\
\hline
\end{tabular}

Values are presented as mean $\pm \mathrm{SD}$ or number $(\%)$.

TNFi, tumor necrosis factor $\alpha$ inhibitor; OR, odds ratio; CI, confidence interval; ESR, erythrocyte sediment rate; CRP, C-reactive protein; HLA, human leukocyte antigen; BMI, body mass index; BASDAI, Bath Ankylosing Spondylitis Disease Activity Index.

${ }^{\text {a }}$ The sacroiliitis grade refers to the sum of each side of the sacroiliitis grade according to the modified New York criteria.

female sex, hip involvement, high CRP, and type of biological drug were associated with drug discontinuation (Table 4). Discontinuation rate was higher in infliximab than etanercept users. For etanercept versus adalimum$\mathrm{ab}$ users, there was no significance in discontinuation in univariable analysis (HR, 2.74; 95\% CI, 1.08 to 6.99; $p$ $=0.035$; adjusted $p=0.107$ ) but there was significance in multivariable analysis (HR, 3.69; 95\% CI, 1.32 to 10.31; $p$ $=0.012$; adjusted $p=0.044)$. There was no statistical sig- nificance between adalimumab versus infliximab users (HR, 2.10; $95 \%$ CI, 0.84 to 5.23; $p=0.110$; adjusted $p$ $=0.279)$. Statistical significant was found in uses for the three TNFi $(p=0.003)$, as shown in Fig. 1.

\section{Reasons for discontinuation}

Table 5 lists the reasons for the discontinuation from the first TNFi. The most common reason for discontinuation was insufficient efficacy (75\%), followed by an 


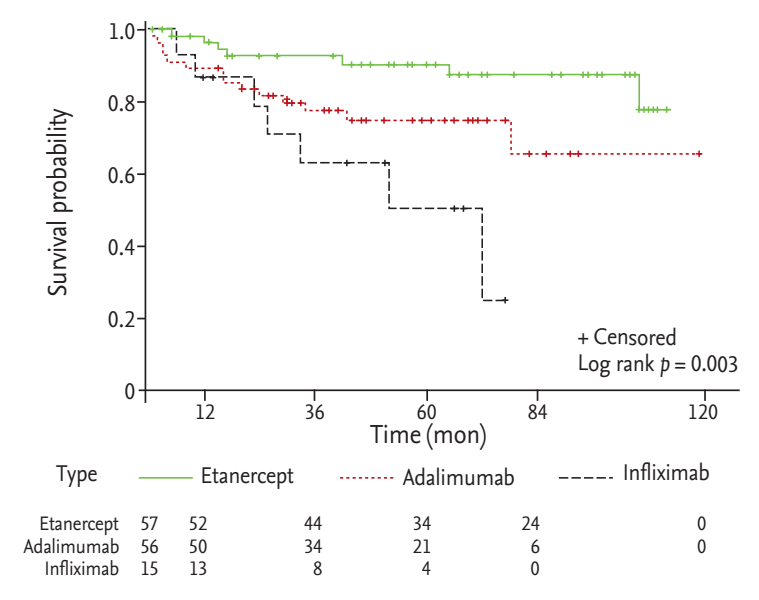

Figure 1. Kaplan-Meier drug survival curves of etanercept, adalimumab, and infliximab as a first tumor necrosis factor $\alpha$ inhibitor discontinuation.

adverse event (14.3\%). Adverse event for one etanercept initiator was injection site reaction. One infliximab initiator developed hypersensitivity characterized by urticaria, dyspnea, and hypotension. Adverse events for two adalimumab initiators included development of psoriasis and herpes infection. Also three patients of each drug discontinued for other reasons. One patient with etanercept switched to adalimumab due to the development of recurrent uveitis. A patient on adalimumab discontinued the biological drug because of intolerance to injection, and one patient on infliximab discontinued the drug for the occurrence of solid cancer.

\section{DISCUSSION}

We investigated drug survival and risk factors for discontinuation of TNFi in patients with AS. From our analysis, early age disease onset, peripheral manifestations, and high acute phase reactant at baseline were associated with future TNFi use. It is similar to the poor prognostic factors associated with AS. A previous study reported that presence of hip arthritis, high ESR, and early disease onset were associated with poor long term outcome of spondyloarthritis [10]. Interestingly, patients with high BMI were more likely to use TNFi than patients with normal BMI. The serum levels of adipokines, such as leptin, TNF- $\alpha$, interleukin 6 have positively correlated with BMI, and concentrations of adipokines are increased in patients with metabolic syndrome [11]. Also, serum leptin levels correlate with the disease activity and associate with presence of syndesmophyte in patients with AS [12,13]. In present study, patients with syndesmophyte had higher BMI than patients without syndesmophyte $\left(24.8 \pm 3.3 \mathrm{~kg} / \mathrm{m}^{2}\right.$ vs. $23.9 \pm 3.5 \mathrm{~kg} / \mathrm{m}^{2}, p=$ $0.021)$. The higher levels of adipokines could be associated with a higher inflammatory burden in obese patients with AS and ultimately result in more TNFi use during the treatment course.

In the current study, the discontinuation of first TNFi was $21.9 \%$, which is similar to the previous studies. Danish DANBIO registry reported that discontinuation rate of TNFi was 37\% (310/842) [14]. A Finnish group reported that the TNFi discontinuation rate was 21\% (49/229) within 2 years of follow-up in their cohort of patients [15]. Norwegian study of 249 patients with AS reported the discontinuation rate of $22.5 \%$ and another study reported the same variable as $14.9 \%$ (77/514) $[16,17]$. Thus, the TNFi discontinuation rate of Korean patients from our study seems to be similar to those with the European patients. Most of the patients who discontinued the first TNFi due to insufficient efficacy showed good response to second TNFi except one patient. This patient was 38 years old male with high disease activity and both hip arthritis. He discontinued the second TNFi due to secondary failure and changed to the third TNFi. Although the symptom was not fully resolved (BASDAI 4), he is maintaining on the third TNFi. In addition, there were two patients who used the third TNFi in the adverse event group. One patient experienced injection site reaction with two TNFis (etanercept, adalimumab). After switching to the third TNFi (infliximab), injection site reaction never developed. The other patient experienced psoriasis with two TNFis (adalimumab, etanercept). The patient did not developed psoriasis with the third TNFi (golimumab).

We found that presence of hip arthritis and high CRP was associated with drug discontinuation. Hip involvement is common manifestation of AS and hip involvement in AS increases the burden and is associated with poor prognosis [18]. Elevated serum level of CRP is a risk factor for radiographic progression in AS [19]. Hip involvement is associated with advanced axial disease, elevated CRP and higher Bath Ankylosing Spondylitis Index scores [20]. Patients with hip arthritis might have 
Table 4. Predictors of first tumor necrosis factor $\alpha$ inhibitor discontinuation

\begin{tabular}{|c|c|c|c|c|c|c|}
\hline \multirow[b]{2}{*}{ Variable } & \multicolumn{3}{|c|}{ Univariable } & \multicolumn{3}{|c|}{ Multivariable } \\
\hline & $\operatorname{HR}(95 \% \mathrm{CI})$ & $p$ value & $\begin{array}{c}\text { Adjusted } \\
p \text { value }\end{array}$ & $\operatorname{HR}(95 \% \mathrm{CI})$ & $p$ value & $\begin{array}{c}\text { Adjusted } \\
p \text { value }\end{array}$ \\
\hline Etanercept & 1.00 (reference) & 0.007 & & 1.00 (reference) & 0.009 & \\
\hline Adalimumab & $2.74(1.08-6.99)$ & 0.035 & 0.107 & $3.69(1.32-10.31)$ & 0.012 & 0.044 \\
\hline Infliximab & $5.77(1.94-17.17)$ & 0.002 & 0.007 & $5.54(1.78-17.22)$ & 0.003 & 0.012 \\
\hline Age & $0.99(0.96-1.03)$ & 0.603 & & - & & \\
\hline Female sex & $2.52(1.07-5.96)$ & 0.036 & & $6.08(2.27-16.27)$ & 0.003 & \\
\hline Disease duration & $1.00(1.00-1.00)$ & 0.870 & & - & & \\
\hline Peripheral arthritis & $1.49(0.71-3.13)$ & 0.295 & & - & & \\
\hline Hip arthritis & $3.08(1.44-6.58)$ & 0.004 & & $2.52(1.08-5.87)$ & 0.033 & \\
\hline Enthesitis & $0.83(0.29-2.38)$ & 0.723 & & - & & \\
\hline Uveitis & $0.36(0.11-1.20)$ & 0.097 & & $0.29(0.08-1.01)$ & 0.052 & \\
\hline Inflammatory bowel disease & $2.67(0.36-19.77)$ & 0.337 & & - & & \\
\hline Syndesmophyte & $0.60(0.23-1.57)$ & 0.296 & & - & & \\
\hline Sacroiliitis grade ${ }^{\mathrm{a}}$ & $0.90(0.70-1.16)$ & 0.431 & & - & & \\
\hline ESR & $1.00(0.99-1.01)$ & 0.677 & & - & & \\
\hline CRP & $1.09(1.00-1.20)$ & 0.046 & & $1.10(1.00-1.21)$ & 0.044 & \\
\hline HLA B-27 & $0.49(0.14-1.70)$ & 0.262 & & - & & \\
\hline BMI & $1.00(0.90-1.11)$ & 0.995 & & - & & \\
\hline Hypertension & $0.92(0.35-2.44)$ & 0.873 & & - & & \\
\hline Diabetes & $1.65(0.49-5.53)$ & 0.415 & & - & & \\
\hline Dyslipidemia & $0.50(0.15-1.65)$ & 0.251 & & - & & \\
\hline Current smoker & $1.91(0.77-4.73)$ & 0.165 & & $1.61(0.64-4.09)$ & 0.315 & \\
\hline BASDAI & $1.08(0.70-1.69)$ & 0.723 & & - & & \\
\hline Prednisolone & $1.04(0.42-2.57)$ & 0.929 & & - & & \\
\hline Sulfasalazine & $1.13(0.34-3.76)$ & 0.839 & & - & & \\
\hline Methotrexate & $0.66(0.20-2.21)$ & 0.499 & & - & & \\
\hline
\end{tabular}

HR, hazard ratio; CI, confidence interval; ESR, erythrocyte sediment rate; CRP, C-reactive protein; HLA, human leukocyte antigen; BMI, body mass index; BASDAI, Bath Ankylosing Spondylitis Disease Activity Index.

${ }^{a}$ The sacroiliitis grade refers to the sum of each side of the sacroiliitis grade according to the modified New York criteria.

Table 5. Reasons for the discontinuation of first tumor necrosis factor $\alpha$ inhibitor discontinuation

\begin{tabular}{lcccc}
\hline Reasons for switching & $\begin{array}{c}\text { Etanercept } \\
(\mathrm{n}=6)\end{array}$ & $\begin{array}{c}\text { Adalimumab } \\
(\mathrm{n}=15)\end{array}$ & $\begin{array}{c}\text { Infliximab } \\
(\mathrm{n}=7)\end{array}$ & $\begin{array}{c}\text { Total } \\
(\mathrm{n}=28)\end{array}$ \\
\hline Insufficient efficacy $^{-12(80.0)}$ & $4(66.7)$ & 4 & $5(71.4)$ & 0 \\
Primary failure $^{\mathrm{a}}$ & 0 & 8 & 5 & 4 \\
Secondary failure $^{\mathrm{b}}$ & 4 & $2(135)$ & 17 \\
Adverse event $^{\text {Others }}$ & $1(16.7)$ & $1(6.7)$ & $1(14.3)$ & $4(14.3)$ \\
\hline
\end{tabular}

Values are presented as number (\%).

${ }^{a}$ Primary failure defined as never achieved meaningful response.

${ }^{\mathrm{b}}$ Secondary failure defined as achieved meaningful response, then lost response during therapy. 
high disease activity during treatment course than patients without hip arthritis, and it could result in more discontinuation of the first TNFi. Risk factors for TNFi discontinuation seem to overlap with poor a prognosis in AS. In addition, female sex was a risk factor for drug discontinuation. It also corresponds with the previous nationwide studies that female sex was a predictor of drug discontinuation in RA [21] and AS [14]. However, male patients with AS have more structural damage than female patients [22]. The reason that the female gender is risk factor for drug discontinuation is unclear. It may be due to females having more peripheral arthritis. In the current study, although occurrence of hip arthritis was not significantly different between males and females, the female patients had more peripheral arthritis than the male ones $(62.5 \%$ vs. $33.9 \%, p=0.027)$. In addition, it may be due to the tendency of women to report poor treatment outcome in subjective parameters $[23,24]$. Female AS patients report worse functioning outcome than males at any level of radiographic damage [22].

In this study, etanercept had the best survival and infliximab had the shortest survival and this corresponds to previous studies. Etanercept had better survival rates than infliximab in patients with RA [21,25] and similar results are found in spondyloarthritis $[7,14,26]$. The number of patients who used infliximab was smaller than other TNFis. Because infliximab injection required intravenous access, patients might have chosen subcutaneous TNFis rather than infliximab. In addition, physician's personal opinion would have affected also. Due to small sample size of infliximab user, there is a possibility that the HR of infliximab about TNFi discontinuation have been overestimated [27].

Development of antidrug antibodies is associated with poor clinical response, the appearance of infusion reactions and the discontinuation of the treatment $[28,29]$. Chimeric monoclonal antibody (infliximab) induces the production of antidrug antibodies more frequently than the fully human drug (adalimumab) and the receptor fusion protein (etanercept). In patients with AS, the formation of antidrug antibody was $0 \%$ in etanercept, whereas infliximab was $20 \%$ to $80 \%$ [30,31]. Comedication of methotrexate reduces the formation of antidrug antibody [32]. Comedication with disease modifying antirheumatic drug (DMARD) was also associated with better retention rate of TNFi in patients with AS [33]. In current study, DMARD use was not related to TNFi discontinuation. In our study, DMARD was not usually administered in the axial form of spondyloarthritis. Most of patients who used DMARD had peripheral manifestations and they discontinued the DMARD rapidly after initiation of TNFi.

There are several limitations in our study. This was a retrospective study from a single tertiary center and study population was small. And data about disease activity parameters such as Bath Ankylosing Spondylitis Functional Index and ASDAS were not available in every patient. However, we found out the real world experience of TNFi use and drug survival in Asian patients with AS. In conclusion, this study document that $21.9 \%$ patients discontinued the first TNFi during the followup period. The TNFi discontinuation rate of Korean patients seems to be similar to those with the European patients. The most common reason for drug discontinuation was inefficacy. In addition, female sex, hip involvement, high levels of CRP, and type of TNFi were associated with drug discontinuation.

\section{KEY MESSAGE}

1. Tumor necrosis factor $\alpha$ inhibitor (TNFi) discontinuation rate of Korean patients with ankylosing spondylitis seems to be similar to those with the European patients.

2. Female sex, hip involvement, C-reactive protein, and the type of TNFi were associated with TNFi discontinuation.

\section{Conflict of interest}

No potential conflict of interest relevant to this article was reported.

\section{Acknowledgments}

This study was financially supported through a grant from the Korea Health Industry Development Institute (HI14C2285).

\section{REFERENCES}

1. Braun J, Bollow M, Neure L, et al. Use of immunohisto- 
logic and in situ hybridization techniques in the examination of sacroiliac joint biopsy specimens from patients with ankylosing spondylitis. Arthritis Rheum 1995;38:499505 .

2. Francois RJ, Neure L, Sieper J, Braun J. Immunohistological examination of open sacroiliac biopsies of patients with ankylosing spondylitis: detection of tumour necrosis factor alpha in two patients with early disease and transforming growth factor beta in three more advanced cases. Ann Rheum Dis 2006;65:713-720.

3. Braun J, Brandt J, Listing J, et al. Treatment of active ankylosing spondylitis with infliximab: a randomized controlled multicentre trial. Lancet 2002;359:1187-1193.

4. Gorman JD, Sack KE, Davis JC Jr. Treatment of ankylos ing spondylitis by inhibition of tumor necrosis factor alpha. N Engl J Med 2002;346:1349-1356.

5. van der Heijde D, Kivitz A, Schiff MH, et al. Efficacy and safety of adalimumab in patients with ankylosing spondylitis: results of a multicenter, randomized, double-blind, placebo-controlled trial. Arthritis Rheum 2006;54:2136-2146.

6. Machado MA, Barbosa MM, Almeida AM, et al. Treatment of ankylosing spondylitis with TNF blockers: a metaanalysis. Rheumatol Int 2013;33:2199-2213.

7. Scire CA, Caporali R, Sarzi-Puttini P, et al. Drug survival of the first course of anti-TNF agents in patients with rheumatoid arthritis and seronegative spondyloarthritis: analysis from the MonitorNet database. Clin Exp Rheumatol 2013;31:857-863.

8. van der Linden S, Valkenburg HA, Cats A. Evaluation of diagnostic criteria for ankylosing spondylitis: a proposal for modification of the New York criteria. Arthritis Rheum 1984;27:361-368.

9. MacKay K, Brophy S, Mack C, Doran M, Calin A. The development and validation of a radiographic grading system for the hip in ankylosing spondylitis: the bath ankylosing spondylitis radiology hip index. J Rheumatol 2000;27:28662872.

10. Amor B, Santos RS, Nahal R, Listrat V, Dougados M. Predictive factors for the longterm outcome of spondyloarthropathies. J Rheumatol 1994;21:1883-1887.

11. Deng Y, Scherer PE. Adipokines as novel biomarkers and regulators of the metabolic syndrome. Ann N Y Acad Sci 2010;1212:E1-E19.

12. Park MC, Lee SW, Choi ST, Park YB, Lee SK. Serum leptin levels correlate with interleukin-6 levels and disease activity in patients with ankylosing spondylitis. Scand J Rheumatol 2007;36:101-106.

13. Kim KJ, Kim JY, Park SJ, et al. Serum leptin levels are associated with the presence of syndesmophytes in male patients with ankylosing spondylitis. Clin Rheumatol 2012;31:1231-1238.

14. Glintborg B, Ostergaard M, Krogh NS, Dreyer L, Kristensen HL, Hetland ML. Predictors of treatment response and drug continuation in 842 patients with ankylosing spondylitis treated with anti-tumour necrosis factor: results from 8 years' surveillance in the Danish nationwide DANBIO registry. Ann Rheum Dis 2010;69:2002-2008.

15. Konttinen L, Tuompo R, Uusitalo T, et al. Anti-TNF therapy in the treatment of ankylosing spondylitis: the Finnish experience. Clin Rheumatol 2007;26:1693-1700.

16. Heiberg MS, Koldingsnes W, Mikkelsen K, et al. The comparative one-year performance of anti-tumor necrosis factor alpha drugs in patients with rheumatoid arthritis, psoriatic arthritis, and ankylosing spondylitis: results from a longitudinal, observational, multicenter study. Arthritis Rheum 2008;59:234-240.

17. Lie E, van der Heijde D, Uhlig T, et al. Effectiveness of switching between TNF inhibitors in ankylosing spondylitis: data from the NOR-DMARD register. Ann Rheum Dis 2011;70:157-163.

18. Vander Cruyssen B, Munoz-Gomariz E, Font P, et al. Hip involvement in ankylosing spondylitis: epidemiology and risk factors associated with hip replacement surgery. Rheumatology (Oxford) 2010;49:73-81.

19. Poddubnyy D, Haibel H, Listing J, et al. Baseline radiographic damage, elevated acute-phase reactant levels, and cigarette smoking status predict spinal radiographic progression in early axial spondylarthritis. Arthritis Rheum 2012;64:1388-1398.

20. Chen HA, Chen CH, Liao HT, et al. Factors associated with radiographic spinal involvement and hip involvement in ankylosing spondylitis. Semin Arthritis Rheum 2011;40:552-558.

21. Neovius M, Arkema EV, Olsson H, et al. Drug survival on TNF inhibitors in patients with rheumatoid arthritis comparison of adalimumab, etanercept and infliximab. Ann Rheum Dis 2015;74:354-360.

22. Lee W, Reveille JD, Davis JC Jr, Learch TJ, Ward MM, Weisman MH. Are there gender differences in severity of ankylosing spondylitis? Results from the PSOAS cohort. Ann Rheum Dis 2007;66:633-638. 
23. Sokka T, Toloza S, Cutolo M, et al. Women, men, and rheumatoid arthritis: analyses of disease activity, disease characteristics, and treatments in the QUEST-RA study. Arthritis Res Ther 2009;11:R7.

24. Katz PP, Criswell LA. Differences in symptom reports between men and women with rheumatoid arthritis. Arthritis Care Res 1996;9:441-448.

25. Marchesoni A, Zaccara E, Gorla R, et al. TNF-alpha antagonist survival rate in a cohort of rheumatoid arthritis patients observed under conditions of standard clinical practice. Ann N Y Acad Sci 2009;1173:837-846.

26. Gulyas K, Bodnar N, Nagy Z, et al. Real-life experience with switching TNF- $\alpha$ inhibitors in ankylosing spondylitis. Eur J Health Econ 2014;15 Suppl 1:S93-S100.

27. Hackshaw A. Small studies: strengths and limitations. Eur Respir J 2008;32:1141-1143.

28. Plasencia C, Pascual-Salcedo D, Nuno L, et al. Influence of immunogenicity on the efficacy of longterm treatment of spondyloarthritis with infliximab. Ann Rheum Dis 2012;71:1955-1960.

29. Maneiro JR, Salgado E, Gomez-Reino JJ. Immunogenicity of monoclonal antibodies against tumor necrosis factor used in chronic immune-mediated inflammatory condi- tions: systematic review and meta-analysis. JAMA Intern Med 2013;173:1416-1428.

30. Arends S, Lebbink HR, Spoorenberg A, et al. The formation of autoantibodies and antibodies to TNF- $\alpha$ blocking agents in relation to clinical response in patients with ankylosing spondylitis. Clin Exp Rheumatol 2010;28:661668.

31. Plasencia C, Pascual-Salcedo D, Garcia-Carazo S, et al. The immunogenicity to the first anti-TNF therapy determines the outcome of switching to a second anti-TNF therapy in spondyloarthritis patients. Arthritis Res Ther 2013;15:R79.

32. Garces S, Demengeot J, Benito-Garcia E. The immunogenicity of anti-TNF therapy in immune-mediated inflammatory diseases: a systematic review of the literature with a meta-analysis. Ann Rheum Dis 2013;72:1947-1955.

33. Lie E, Kristensen LE, Forsblad-d'Elia H, et al. The effect of comedication with conventional synthetic disease modifying antirheumatic drugs on TNF inhibitor drug survival in patients with ankylosing spondylitis and undifferentiated spondyloarthritis: results from a nationwide prospective study. Ann Rheum Dis 2015;74:970-978. 